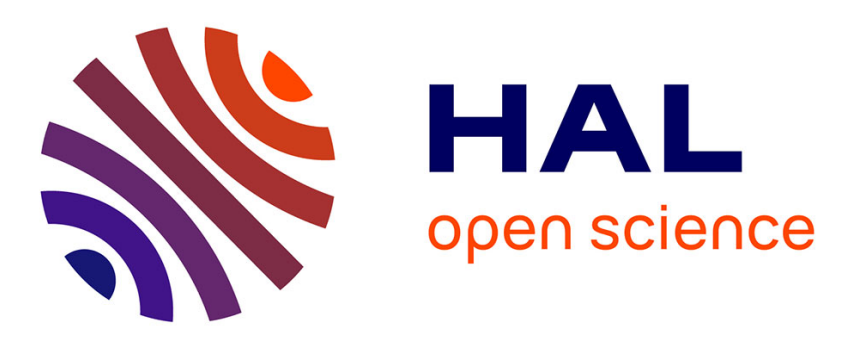

\title{
Enjeux des politiques linguistiques: pratiques et comportements langagiers multilingues dans un pays monolingue
}

\author{
Shahzaman Haque
}

\section{- To cite this version:}

Shahzaman Haque. Enjeux des politiques linguistiques: pratiques et comportements langagiers multilingues dans un pays monolingue. Maria Iliescu; Heidi Siller-Runggaldier; Paul Danler. Actes du XXVème Congrès International de Linguistique et de Philologie Romanes, Tome 1, Walter de Gruyter, pp.163-171, 2010, 978-3-11-023193-9. hal-02769580

\section{HAL Id: hal-02769580 \\ https://hal-inalco.archives-ouvertes.fr/hal-02769580}

Submitted on 4 Jun 2020

HAL is a multi-disciplinary open access archive for the deposit and dissemination of scientific research documents, whether they are published or not. The documents may come from teaching and research institutions in France or abroad, or from public or private research centers.
L'archive ouverte pluridisciplinaire HAL, est destinée au dépôt et à la diffusion de documents scientifiques de niveau recherche, publiés ou non, émanant des établissements d'enseignement et de recherche français ou étrangers, des laboratoires publics ou privés. 


\section{Shahzaman Haque}

\section{Enjeux des politiques linguistiques: pratiques et comportements langagiers multilingues dans un pays monolingue}

\section{Introduction}

Cet article propose un aperçu de la situation sociolinguistique que peut rencontrer une famille indienne migrante en France. Cette étude est basée sur le comportement linguistique d'une famille, c'est-à-dire, les choix de langue au sein d'un foyer et quels sont les statuts de chacune de ces langues dans celui-ci.

Plusieurs méthodes ont été employées pour mener cette recherche qui a pour objectif d'analyser l'emploi de chacune des langues, ainsi que les conditions expliquant le maintien ou la disparition possible de l'une de ces langues dans le répertoire des usagers. Il est proposé comme hypothèse que l'alternance codique est un lieu privilégié d'observation pour répondre à ces questions. Les données utilisées sont trois conversations enregistrées au sein de cette famille plurilingue qui parle l'haryanvi, l'hindi, l'anglais et le français, et cela au cours de deux ans. Ces trois conversations ont été retranscrites selon les principes de l'analyse conversationnelle et forment trois corpus. Des entretiens et des questionnaires ont apporté des informations supplémentaires au sujet de chacun des membres de la famille.

\section{Politique linguistique nationale}

Nous faisons l'hypothèse que la politique linguistique menée par un pays a un effet direct sur les représentations linguistiques et les comportements langagiers des locuteurs. La France, qui a une politique monolingue par opposition à l'Inde, démontre une certaine difficulté à accepter les langues des migrants. En effet, l'absence de politique spécifique visant au maintien des langues est un indice de ce manque de prise en considération des aspects linguistiques de la migration. Le français est en quelque sorte survalorisé au détriment des autres langues, et les locuteurs français indigènes monolingues considèrent comme 〈allant de soi〉 d'avoir accès à l'ensemble des connaissances grâce à une langue unique. Ils ne ressentent donc pas le besoin d'apprendre une autre langue pour enrichir leur savoir. Cette idéologie monolingue a pour conséquence que de nombreux migrants optent pour le français comme langue unique de communication au détriment de leur(s) langue(s) native(s). Etant un pays plurilingue et beaucoup moins centralisé que la France, l'Inde 
mène une politique linguistique différente dans chaque Etat, ce qui a pour résultat vingtdeux langues officielles et un millier de langues premières. Les répertoires indiens sont donc plurilingues et le changement de langue s'effectue en fonction des interlocuteurs.

\section{Politique linguistique familiale}

Lors de la présentation de cette communication au colloque, le terme spolitique linguistique familiale> a été discuté et remis en question par plusieurs congressistes qui préféraient le terme «stratégie familiale». Cependant, le terme «stratégie〉 n'est pas adapté lorsqu'il s'agit de la pratique de langues, quelles qu'elles soient, au sein d'une famille.

Le terme 〈stratégie linguistique〉 s'emploie lorsqu'un individu qui souhaite apprendre une seconde langue choisit les formes d'apprentissage les plus adaptées à ses propres capacités et ses propres objectifs, une compétence linguistique. Il met ainsi ses connaissances au service de l'apprentissage de la langue et se donne alors tous les moyens pour réussir. Tout apprentissage et toute acquisition d'une langue dans le propre milieu de l'individu font partie de sa stratégie décrite par Grosjean (1993) comme une «stratégie cognitive, sociale et linguistique».

Une politique linguistique sous-entend la mise en cause d'un groupe social tel que le groupe familial que nous prendrons comme exemple. Le ou les chefs de la famille opère(nt) un choix vis-à-vis des langues que le reste des membres de la famille doivent apprendre et doivent employer dans certaines sphères, défini par Martine Dreyfus (1996) comme «des règles et des contraintes». $\mathrm{Il}(\mathrm{s})$ exprime(nt) ainsi sa/leur volonté aux autres mais ne peut/peuvent garantir des résultats obtenus et de l'investissement des membres visés par sa/leur politique. De la même manière que la politique linguistique nationale est menée par les instances gouvernementales, une politique linguistique est menée dans chaque foyer par le(s) chef(s) de la famille qui prend (prennent) des décisions pour l'ensemble des membres de la famille. On peut alors citer Christine Deprez (1996) qui emploie le terme spolitique linguistique familiale> lorsque la famille crée sa propre gérance de la langue.

Néanmoins, de même qu'il existe des situations où certains des membres s'opposent et résistent à la politique linguistique familiale, il est également possible de s'opposer à la politique linguistique nationale. A partir du moment où l'individu possède des compétences suffisantes pour s'exprimer dans une langue autre que la langue première, il peut choisir d'employer telle ou telle langue. Alors, la politique linguistique familiale peut, elle aussi venir contrer la politique linguistique nationale. D'après Christine Deprez (1996), «dès que l'on aborde la sphère de la vie privée, les décisions prises 〈d'en haut perdent de leur impact». Elle cite l'exemple de l'interdiction de l'usage du Catalan sous Franco, interdiction qui n'a jamais réussi à franchir les frontières des foyers. Les Catalans ont continué à parler leur langue et à transmettre celle-ci à leurs enfants malgré la politique linguistique nationale. Ceci met en évidence le fait que chaque famille mène une politique linguistique différente qui peut être ou non conforme avec la politique linguistique nationale. 
C'est donc une politique linguistique familiale qui est mise en place dans le foyer pris comme exemple dans cette étude. En effet, les parents ont joué un rôle majeur dans l'usage des langues, non seulement à l'intérieur de la maison mais aussi dans la société monolingue où ils vivent. Nous verrons plus tard comment et pourquoi certaines décisions ont été prises par les parents et ont largement influencé le comportement linguistique des enfants sans pour autant le déterminer.

La famille se compose de deux enfants et deux adultes, une fille aînée et un fils cadet et leurs parents qui vivaient ensemble à Grenoble au moment du recueil des données. Les deux parents, des migrants de l'Inde, ont tout en commun y compris leur parcours linguistique et les langues apprises à l'école. Etant donné que le père a beaucoup voyagé en Inde, il maîtrise deux ou trois langues de plus que sa femme. Sa migration en France est un pur hasard, comme c'est le cas des familles migrantes qui n'ont pas choisi leur destination au départ. Par conséquent, il n'avait aucune connaissance de la langue française avant de s'installer. Son apprentissage du français s'est réalisé 〈sur le tas〉 et il n'a jamais suivi de cours. Après avoir vécu huit ans en France, il s'est marié avec une Indienne appartenant à la même communauté (même Etat: Haryana, même langue première: Haryanvi) qui est venue le rejoindre sur place six mois plus tard. L'arrivée de celle-ci n'a pas, à première vue, modifié la place des langues au sein du noyau familial. Ils ont continué à parler l'haryanvi / l'hindi entre eux à la maison et KAM a continué à utiliser le français comme langue pour communiquer à l'extérieur du foyer. SUN n'avait, elle non plus, aucune connaissance de la langue française à son arrivée. Elle a pris immédiatement des cours particuliers, ensuite elle s'est inscrite au CUEF (Centre Universitaire d'Etudes Françaises) pendant deux ans afin d'obtenir l'autorisation de s'inscrire à l'université pour faire une licence en anglais. Lorsque les enfants ont grandi, la situation linguistique de la famille a connu de nombreux bouleversements puisque les enfants ont été scolarisés sur place. Notons que les langues premières des parents, qui ont aussi une bonne compétence en anglais et en français, sont l'haryanvi et l'hindi. En ce qui concerne les enfants, leurs langues premières sont le français et l'haryanvi bien qu'ils aient été scolarisés en anglais et qu'ils aient des connaissances en hindi.

Corpus I: Politique linguistique familiale conflictuelle

Dans le premier corpus enregistré le 3 juillet 2004, le père de la famille, KAM réside en France depuis 22 ans et SUN, sa femme depuis 14 ans, leurs deux enfants sont nés et ont été scolarisés en France et sont âgés de 10 (le fils: ANM) et 13 (la fille: IPS) ans. La durée d'enregistrement est d'une heure et vingt-cinq minutes et le lieu est l'appartement de la famille. 


\section{Extrait I}

$\begin{array}{lll}\text { KAM } & : & \begin{array}{l}\text { Joke aate hain koi India } k e ? \\ \text { \%fr tu connais une blague indienne? }\end{array} \\ \text { ANM } & : & \begin{array}{l}\text { Non. } \\ \text { KAM }\end{array} \\ \text { ANM } & : & \begin{array}{l}\text { Hindi joke? } \\ \text { \%fr la blague en hindi? }\end{array} \\ \text { KAM } & : & \begin{array}{l}\text { Non. } \\ \text { French aata hai? }\end{array} \\ \text { ANM } & : & \text { \%on tu connais le français? } \\ \text { KAM } & : & \begin{array}{l}\text { English joke aata hai? } \\ \text { \%fr tu connais la blague en anglais? }\end{array} \\ \text { ANM } & : & \begin{array}{l}\text { Non } \\ \text { Kahin aata hai to go and sleep. }\end{array} \\ \text { KAM } & : & \text { \%fr si tu ne sais pas, va te coucher. }\end{array}$

Dans cette discussion, KAM le père essaie de faire raconter à son fils une blague, et pour cela il utilise l'hindi, mais son fils, nettement récalcitrant, fait une alternance divergente en utilisant une autre langue, le français, pour répondre à la négative. Il effectue donc une double négation par le contenu et la forme de sa réponse.

Le père met en avant son caractère inflexible en maintenant l'hindi pour exprimer son autorité vis-à-vis de son fils au détriment de la communication.

\section{Extrait II}

Un deuxième extrait du même corpus, où les trois membres de la famille conversent en anglais et en français, souligne l'importance de ces deux langues dans leurs pratiques langagières.

\begin{tabular}{|c|c|c|}
\hline KAM & : & $\begin{array}{l}\text { (s'adressant à ANM) And what do you think? } \\
\% \text { fr et qu'est-ce que t'en penses? }\end{array}$ \\
\hline ANM & : & $\begin{array}{l}\text { Why are you asking all the questions to me? } \\
\% \text { fr pourquoi vous me posez toutes les questions? }\end{array}$ \\
\hline SUN & : & $\begin{array}{l}\text { Because you are the star, vedette cette soir. } \\
\% \text { fr parce que tu es la star, vedette ce soir. }\end{array}$ \\
\hline ANM & : & $\begin{array}{l}\text { Non, pourquoi tu demandes pas à maman? } \\
\text { *fr non, pourquoi tu ne demandes pas à maman? }\end{array}$ \\
\hline KAM & : & $\begin{array}{l}\text { Parce qu'elle regarde pas le foot. } \\
\text { *fr parce qu'elle ne regarde pas le foot. }\end{array}$ \\
\hline ANM & & Pose la question à Maman. \\
\hline SUN & & $\begin{array}{l}\text { Bah, je peux dire que le football, j'aime pas. } \\
* \text { fr bah, je peux dire que le football, je n'aime pas }\end{array}$ \\
\hline
\end{tabular}

Dans cet extrait il est possible de remarquer la présence de langues dans le foyer qui ne sont pas les langues premières des parents, en particulier le français qui revient 
systématiquement malgré les tentatives des parents de parler dans une autre langue (l'anglais). Avec ces quelques interactions, il est impossible de localiser l'origine de cette famille migrante et l'hindi et l'haryanvi semblent être relégués au second plan.

Dans une telle situation où plusieurs langues sont parlées par les membres de la famille, le microcosme familial plurilingue peut devenir une scène de conflit car chaque membre s'exprime à travers la langue qu'il maitrise le mieux ou qu'il préfère. Ainsi, ne pas avoir une langue commune pour effectuer une conversation fluide peut aboutir à une situation conflictuelle et cela peut provoquer de la frustration et de la colère parmi les membres de la famille. La discussion entre le père et le fils illustre cette forme de communication inaboutie et frustrante. Cette alternance codique, ce glissement permanent entre plusieurs langues parmi les membres de la famille, crée un microcosme linguistique. La communication est alors ‘fragilisée〉 lorsque l'un des deux partis refuse de céder face à la pratique langagière de l'autre soit par volonté soit par incapacité linguistique. Cette famille s'est retrouvée confrontée à cette situation puisque les parents se sont rendus compte de l'incapacité et du manque de volonté de leurs enfants pour parler en hindi, et ils ont donc décidé de scolariser leurs enfants en Inde pour qu'ils puissent apprendre les langues indiennes et obtenir de nouvelles notions culturelles.

Corpus II: Réintégration linguistique et culturelle au pays d'origine

Le deuxième corpus, a été enregistré le 25 décembre 2005 et sa durée est d'une heure et quarante-cinq minutes. Il a eu lieu dans l'appartement en présence de KAM, SUN, ANM et IPS. La famille est de retour en France après avoir passé six mois en Inde durant lesquels les enfants étaient scolarisés sur place. Un changement radical s'est opéré en ce qui concerne leur compétence dans les langues indiennes.

\section{Extrait I}

Ceci est un extrait de ce corpus où le père et le fils dialoguent tous les deux en hindi, sans que ce choix linguistique soit problématique:

\begin{tabular}{|c|c|c|}
\hline KAM & : & $\begin{array}{l}\text { Tu kiyon baar baar isse bahas karta hai Anmol? } \\
\% \text { fr pourquoi tu te disputes avec elle Anmol? }\end{array}$ \\
\hline ANM & : & $\begin{array}{l}\text { Bahas nahin kar raha. } \\
\% \text { fr je ne me dispute pas. }\end{array}$ \\
\hline KAM & : & $\begin{array}{l}\text { Aur kiya, ke mere ziyada hain, yeh woh, tu kiyon baat karta hai. } \\
\% \text { fr et quoi encore? 〈J'ai les meilleures〉 et d'une, et de deux. } \\
\text { Pourquoi tu parles? }\end{array}$ \\
\hline ANM & : & $\begin{array}{l}\text { Yeh bolti hai. } \\
\% \text { fr c'est elle qui parle. }\end{array}$ \\
\hline KAM & : & $\begin{array}{l}\text { To bolne do usko. } \\
\text { \%fr laisse-la parler. }\end{array}$ \\
\hline
\end{tabular}


Le père est maintenant assuré que s'il engage une discussion en hindi, c'est-à-dire sa deuxième langue, son fils sera capable de maintenir l'échange dans la même langue puisqu'il a vécu et été scolarisé en Inde.

\section{Extrait II}

L'exemple suivant met en évidence la facilité que notre locuteur possède à produire une alternance codique avec trois langues différentes:

ANM : Ok...meri english isse achchi se hai, kiyonki mein Europole mein, moi j'suis dans le groupe quatre, dès que j'suis entré en sixième, test jab pass hua tha na to directly mein group four mein aaya, highest group mein, aur Ipshita ne jab paas kiya tha na to group two mein aayi thi, sab se chota group, iska matlab ke mera english Ipshita se achcha hai.

\%fr mon anglais est meilleur que le sien, parce que je suis à Europole, moi je suis dans le groupe quatre, dès que je suis entré en sixième, quand j'ai passé l'épreuve, ils m'ont mis directement dans le groupe quatre, le groupe le plus fort, et quand Ipshita a passé cette épreuve, elle avait été mise dans le groupe deux, le groupe le plus faible, cela veut dire que mon anglais est meilleur que celui d'Ipshita.

Cette longue intervention de ANM qui passe librement d'une langue à l'autre montre sa 〈souplesse` avec chacune des langues utilisées. Cette alternance codique qui est naturelle de sa part ne semble pas avoir de fonction puisque le choix de langue ne sert pas à transmettre un méta-message. ANM utilise chacune d'entre elles dans le seul but de s'exprimer car elles ont perdu toute connotation extérieure. Il met en avant sa propre spolitique linguistique> qui pourrait être caractérisée comme une absence de reconnaissance de la politique linguistique imposée par ses parents et du statut social de chacune des langues, en juxtaposant ces trois langues (le français, l'anglais et l'hindi), il crée alors un compromis entre la politique linguistique familiale et la politique linguistique nationale.

Une nouvelle situation s'est donc instaurée dans la famille comme le montrent les deux exemples précédents. La communication entre les membres de celle-ci se déroule pacifiquement et la tension s'est beaucoup apaisée grâce au séjour des enfants en Inde qui leur a permis d'approfondir leur connaissance des langues indiennes. Le choix de l'hindi n'est plus problématique. Néanmoins, du point de vue des parents, l'hindi, l'haryanvi et l'anglais sont plus importants que le français et ils souhaitent que leurs enfants parviennent à les maîtriser. 
Corpus III: Domination des langues premières

Le troisième et le dernier corpus, enregistré le 15 juin 2006 et dont la durée est de quarante-quatre minutes, s'est déroulé une fois encore dans l'appartement de la famille en présence de tous les membres de celle-ci. Cette fois-ci, les enfants sont revenus en France pour les vacances après avoir passé un an et demi en Inde.

L'exemple tiré de ce corpus souligne à quel point l'utilisation de l'hindi s'est imposée au sein de la famille.

\begin{tabular}{|c|c|c|}
\hline SUN & & $\begin{array}{l}\text { Achcha achcha, professional ho gaye tum. } \\
\% \text { fr ah bon, ah bon, tu es devenu professionnel. }\end{array}$ \\
\hline ANM & : & $\begin{array}{l}\text { Nahin mujhe jo bhi khela do, sab khelta hun. } \\
\% \text { fr non, on peut me faire pratiquer n'importe quel sport, je les } \\
\text { pratique tous. }\end{array}$ \\
\hline KAM & : & $\begin{array}{l}\text { Abki baar jayega na, to gymnastic shru kar na, you can do good } \\
\text { gymnastic. } \\
\text { \%fr quand tu retourneras (en Inde) cette fois, commence à faire } \\
\text { de la gymnastique, tu peux être très bon en gymnastique. }\end{array}$ \\
\hline IPS & : & $\begin{array}{l}\text { Hai hi nahin school mein. } \\
\text { \%fr il n'y en a pas à l'école. }\end{array}$ \\
\hline KAM & : & $\begin{array}{l}\text { Try karna, you can be good in gymnastics. } \\
\% \text { fr tu dois essayer, tu peux être bon en gymnastique. }\end{array}$ \\
\hline ANM & : & $\begin{array}{l}\text { Meine yehan par kiya tha char saal phir... } \\
\% \text { fr je l'ai pratiqué ici pendant quatre ans puis... }\end{array}$ \\
\hline IPS & : & $\begin{array}{l}\text { Phir? } \\
\% \text { fr et puis? }\end{array}$ \\
\hline ANM & : & $\begin{array}{l}\text { Band kar diya. } \\
\% \text { fr j'ai arrêté. }\end{array}$ \\
\hline KAM & : & $\begin{array}{l}\text { Jana shru kar de, to kiya hua. } \\
\% \text { fr tu peux recommencer, ce n'est pas grave. }\end{array}$ \\
\hline ANM & : & $\begin{array}{l}\text { Association achchi nahin thi isliyé. } \\
\% \text { fr il n'y avait pas de bonne association sportive. }\end{array}$ \\
\hline KAM & : & $\begin{array}{l}\text { Koi baat nahin hai, jahan association achchi hogi wohan kar } \\
\text { lenge. }\end{array}$ \\
\hline
\end{tabular}

Dans cet acte de parole, tous les membres de la famille sans exception communiquent en hindi ce qui n'était pas concevable deux ans auparavant. Il est possible de remarquer l'emploi de mots appartenant au lexique anglais qui sont des exemples de mélange codique dénué de fonction, puisqu'il s'agit ici de mélange codique institutionnalisé du fait du long contact entre la langue hindi et la langue anglaise, suite à la colonisation de l'Inde par les Britanniques. De nombreux termes lexicaux anglais ont remplacé leurs équivalents dans les langues indiennes ou se sont intégrés dans l'usage quotidien de l'hindi. 


\section{Conclusion}

Sachant que les enfants doivent rentrer définitivement un jour en France, on peut se demander sur quelles démarches seront prises par les parents pour leur réimmersion. De même quelle sera la politique menée dans le foyer pour un choix de langue non conflictuelle? Le danger est que cette famille revienne à son point de départ en ce qui concerne la domination du français et le conflit linguistique avant le séjour en Inde. Il est probable qu'une telle politique linguistique menée par la famille et par le pays d'accueil crée des migrants tiraillés entre deux cultures et au moins deux langues.

Nous nous interrogeons alors sur la manière dont, dans une famille migrée, la culture et la langue d'origine peuvent interagir avec la culture et la langue du pays d'accueil, et si l'assimilation et la maîtrise d'une langue provoquent systématiquement la disparition d'une autre. Dans le cadre de cette famille indienne migrée, une interrogation subsiste au sujet du comportement langagier futur des enfants, bien intégrés dans leur deux cultures et ayant deux langues natives. Feront-il un mélange de code systématique en créant leur propre variété de langue? Comment construiront-ils leurs énoncés tout en restant en accord avec leur identité plurielle?

\section{Bibliographie}

Auer, Peter (1998): Code-Switching in Conversation: Language, Interaction and Identity. New York: Routledge.

Billiez, Jacqueline (1995): La langue comme marqueur d'identité. In: Revue Européenne des Migrations Internationales. Vol. I, n², 95-105.

Bloomfield, Leonard (1933): Language. New York: Holt, Rinehart and Winston.

Bourdieu, Pierre (1982): Ce que parler veut dire. Paris: Fayard.

Canut, Cécile / Caubet, Dominique (2001): Comment les langues se mélangent, Codeswitching en Francophonie. Paris: L'Harmattan.

Dabène, Louise / Billiez, Jacqueline (1984): Recherches sur la situation sociolinguistique des jeunes issus de l'immigration (Première Partie). Centre de Didactique des Langues, Grenoble III.

- (1986): Code-Switching in the speech of adolescents born of immigrant parents. In: Studies in Second language acquisition 8, 309-325.

Deprez, Christine (1994): Les enfants bilingues: langues et familles. Paris: Didier.

- (1996): Politique linguistique familiale: le rôle des femmes. In: Juillard, C. / Calvet, Louis-Jean (edd.): Politiques linguistiques, mythes et réalités (les). Actes des premières Journées scientifiques $\mathrm{du}$ réseau «Sociolinguistique et dynamique des langues» de l'AUF, Dakar (Sénégal), 16-18 décembre 1995. AUF: Fiches du Monde Arabe, 155-161.

Dreyfus, Martine (1996): Politiques linguistiques familiales et individuelles: quels modèles? In: Juillard, C. / Calvet, Louis-Jean (edd.): Politiques linguistiques, mythes et réalités (les). Actes des premières Journées scientifiques du réseau «Sociolinguistique et dynamique des langues» de l’AUF, Dakar (Sénégal), 16-18 décembre 1995. AUF: Fiches du Monde Arabe, 175-181.

Franceschini, Rita (1998): Code-Switching and the notion of code in linguistics: Proposal for a dual focus model. In: Code-Switching in Conversation: Language, Interaction and Identity, 51-75. 
Grosjean, François (1984): Le bilinguisme: vivre avec deux langues. In: Tranel 7.

- (1993): Le bilinguisme et le biculturalisme: essai de définition. In: Tranel.

Haque, Shahzaman (2006): Choix ou alternance des langues au sein de la famille indienne immigrée en France. Memoire de Master non publié. Sous la direction de Jacqueline Billiez. Université Stendhal. Grenoble, III.

Heller, Monica (1988): Codeswitching: Anthropological and Sociolinguistic Perspectives. Berlin / New York / Amsterdam: Mouton de Gruyter.

Leclerc, Jacques (2005): Inde. In: L'aménagement linguistique dans le monde. www.tlfq.ulaval.ca/axl/asie/inde-1Union.htm (2006 04 18).

Lüdi, Georges / Py, Bernard (1986): Etre Bilingue. Berne: Peter Lang.

- (2003): Etre Bilingue. Berne: Peter Lang.

Rampton, Ben (1995): Crossing: Language and ethnicity among adolescents. New York: Longman. 
